\title{
Melanoma-Nevus Discrimination Based on Image Statistics in Few Spectral Channels
}

\author{
Aivars Lorencs ${ }^{1}$, Juris Sinica-Sinavskis ${ }^{1}$, Dainis Jakovels ${ }^{2}$, Ints Mednieks ${ }^{1}$ \\ ${ }^{1}$ Institute of Electronics and Computer Science, \\ Dzerbenes St. 14, LV-1006 Riga, Latvia \\ ${ }^{2}$ Biophotonics Laboratory, Institute of Atomic Physics and Spectroscopy, University of Latvia, \\ Raina Blvd. 19, LV-1586 Riga, Latvia \\ mednieks@edi.lv
}

\begin{abstract}
The purpose of this paper is to offer a method for discrimination of cutaneous melanoma from benign nevus, founded on analysis of skin lesion image. At the core of method is calculation of mean and standard deviation of pixel optical density values for a few narrow spectral bands. Calculated values are compared with discriminating thresholds derived from a set of images of benign nevi and melanomas with known diagnosis. Classification is done applying weighted majority rule to results of thresholding. Verification against the available multispectral images of 32 melanomas and 94 benign nevi has shown that the method using three spectral bands provided zero false negative and four false positive melanoma detections. The proposed classifier is characterized by high sensitivity and specificity concerning statistical point estimates whereas its possible technical implementation is fairly simple. The proposed method may be instrumental for designing low cost diagnostic equipment to be used in primary care that is important for early detection of cutaneous melanoma.
\end{abstract}

Index Terms-Image classification; biomedical optical imaging; multispectral imaging; melanoma detection.

\section{INTRODUCTION}

Skin melanoma is the most dangerous type of skin cancer. It was estimated that 76100 new cases will be diagnosed and 9710 people will die of melanoma in United States in 2014 [1]. Melanoma accounts for less than $2 \%$ of all skin cancer cases, but it causes vast majority of skin cancer deaths.

Significant increase in melanoma incidence (from 5.1 in 1998 to 7.8 new cases per 100000 inhabitants in 2008) was observed in Latvia and similar trend was observed in other European countries [2] where the estimated incidence of melanoma (measured per 100000 inhabitants) varies form 19.1 in Northern Europe (NE) to 6.1 in Central and Easter Europe (CEE) with average value of 11.2 in whole Europe [3]. Melanoma mortality rates are much more similar -2.3 in CEE, 3.0 in NE and 2.3 in whole Europe. The differences in estimates of melanoma incidence and mortality are likely to be related to deficient early diagnostics in Central and Eastern Europe [3], [4].

Melanomas often start as small, mole-like growths that increase in size and may change colour [1]. Therefore, non-

Manuscript received 30 April, 2015; accepted 7 March, 2016.

This work was supported by the European Social Fund within project "Innovative biomedical image acquisition processing technologies (InBiT)" (No.2013/0009/1DP/1.1.1.2.0/ 13/APIA/VIAA/014) invasive, fast methods for primary diagnostics of pigmented skin lesions are highly demanded by clinicians [5]. A simple $\mathrm{ABCD}$ rule is often used in clinical practice for visual assessment of melanoma [6] where A stands for asymmetrical shape, B for border irregularity, $\mathrm{C}$ for colour and tonal variation, and $\mathrm{D}$ for differential structures. As this approach is largely subjective, there are attempts to supplement it with calculation of additional quantitative features that can be used for development of viable decision support systems [7].

Diagnostics of skin melanoma based on hyperspectral or multispectral imaging is an alternative direction which has lately attracted attention of a number of scientific groups [8]-[16]. Various data processing algorithms of multispectral image cubes are used, e.g. for extraction of morphological and reflection related parameters [10], [11], evaluation of melanoma thickness [12], spectral angle calculation between normal skin and lesion [13], regression analysis for assessment of skin chromophores [14], [15], combination of several spectral channels [16] and application of artificial neural networks [17]. Spectral features of skin lesions are recognized as a valuable additional source of information and are considered helpful for diagnostics, thus supplementing the commonly used $\mathrm{ABCD}$ rule with more advanced assessment of colour and tonal variation. Several spectral imaging approaches have been implemented in commercial devices. Two of the most popular multispectral dermoscopes are MelaFind [18] and SIAscope [19]. MelaFind (Electro-Optical Sciences, Irvington, NY, USA) extracts data on specific features from 10 spectral images in $430 \mathrm{~nm}-950 \mathrm{~nm}$ range for diagnosis of melanoma and has demonstrated $95 \%$ to $100 \%$ sensitivity and $70 \%$ to $85 \%$ specificity [18]. SIAscope (Astron Clinica, Cambridge, UK) analyses the distribution of a lesion's position and quantity of chromophores from 8 spectral bands in $400 \mathrm{~nm}-1000 \mathrm{~nm}$ range and has shown $83 \%$ sensitivity and $80 \%$ specificity [19]. However, spectral imagers are still not widely used to analyse skin lesions in medical practice due to their high cost and also insufficient clinical trials of their diagnostic methods.

Analysis of skin lesion images and chromophores spectra has led to development of diagnostic methods which employ the most informative spectral features that may indicate presence of melanoma. Results of a clinical trial published 
by Diebele et al. [16] show that melanomas and common nevi can be discriminated with high sensitivity (94\%) and specificity $(89 \%)$ using optical density values from only 3 $(540 \mathrm{~nm}, 650 \mathrm{~nm}$ and $950 \mathrm{~nm})$ narrow spectral bands. Other study, Quinzán et al. [20] searched for optimal number of bands in narrow band spectral imaging for melanoma diagnosis and concluded that the best results (100\% sensitivity and $72 \%$ specificity) were obtained when 9 to 19 bands out of 55 were used. Dhawan et al. [21] has demonstrated multispectral transilluminator Nevoscope for melanoma detection by images in 3 spectral bands at $510 \mathrm{~nm}, 560 \mathrm{~nm}$ and $610 \mathrm{~nm}$. Kapsokalyvas et al. [22] has reported on the development of a polarization multispectral dermoscope for in vivo imaging of skin lesions. Three spectral bands $(470 \mathrm{~nm}, 530 \mathrm{~nm}$ and $625 \mathrm{~nm}$ ) were used for data extraction on skin chromophore content that can be used for detection of pathologies.

On the other hand, employment of not only mean value but also standard deviation of reflectance values was suggested by Farina et al. [9]. Application of both the reflectance mean values and standard deviations to enable employment of small number of spectral bands was suggested by Lorencs et al. [23] for classification of multispectral image fragments. Statistics of pixel values were calculated separately for each spectral band. Limiting the number of the used spectral bands to only few (e.g. 3) would allow to replace hyperspectral or multispectral cameras with much simpler hardware, shorten the data acquisition procedure, and reduce the amount of data to be processed.

Lorencs et al. [23] suggested also using this approach for classification of skin lesions in biomedical image processing. Current paper is focused on the attempt to apply such simplified approach to the task of distinguishing melanomas from benign nevi to pave the way towards development of low cost diagnostic equipment that could be used by primary care physicians and help them to decide whether further investigation of the skin lesion should be prescribed.

\section{IMAGE ACQUISITION AND PREPROCESSING}

The diffuse reflectance spectral imaging data of pigmented skin lesions used in this study were acquired during the years 2010 and 2011 in three Riga clinics under a protocol approved by the local ethics committee [15]. Data of 126 cases (32 melanomas from 31 patient and 94 nonmalignant pigmented lesions, each from a different patient) were pre-processed and made available for the study by the Institute of Atomic Physics and Spectroscopy of the University of Latvia. All cases of melanoma were diagnosed in Latvian Oncology Center and confirmed by experienced dermatologists. The measurements were taken from patients in supine position, in order to minimize the motion artefacts.

The spectral image acquisition system used for acquiring images was built around the multispectral imaging camera Nuance EX (Cambridge Research \& Instrumentation, USA) comprising a liquid crystal tunable filter and built-in polariser. Home-made stabilized halogen lamp ring was used as a light source, with diffuser and polariser in front of it. Cross polarization set-up of the light source polariser and built-in polariser of the camera was used to reduce interference of specular reflectance from skin surface [24]. The system operated in the $450 \mathrm{~nm}-950 \mathrm{~nm}$ range, sequentially acquiring spectral images with a $10 \mathrm{~nm}$ step and spectral resolution (FWHM) $\sim 15 \mathrm{~nm}$. Resulting image cube consisted of 51 images with spatial resolution $\sim 0.05 \times 0.05$ mm per pixel.

Pre-processing of the data included calculation of optical density values, registration of images for motion artefact removal and segmentation of melanoma and nevus objects. White reference $\left(I_{0}\right)$ was used to convert diffuse reflectance intensity $(I)$ value to optical density (OD)

$$
\mathrm{OD}(\lambda)=-\log _{10}\left[I(\lambda) / I_{0}(\lambda)\right]
$$

Home-made white paper plate attached to the skin surface was used for white reference measurements and further spectrally corrected according to white reference tile WS-2 (Avantes, Netherlands). Removal of motion artefacts was performed using MultiStackReg and Image Stabilizer plugins in Image J program.

Optical density mean value and standard deviation was calculated for each pigmented skin lesion manually segmented in the image. Segmentation was performed by marking a polygon at the outer boundary of a lesion. Manual segmentation was used in this study intentionally to exclude potential segmentation errors from processing and focus on investigation of the discrimination approach of melanoma lesions from common nevi lesions. Segmentation of skin lesions is a separate quite sophisticated task (see [25]) not discussed in this paper.

\section{Processing MethoD}

The set of 32 optical density images of melanomas and 94 images of nevi obtained in a way described above was used for the development of the classifier of melanomas and nevi. Images were acquired in 51 spectral bands with equal bandwidths, numbered from 1 to 51 and centred around the wavelengths $\lambda_{1}=450 \mathrm{~nm}, \lambda_{2}=460 \mathrm{~nm}, \ldots, \lambda_{51}=950 \mathrm{~nm}$.

To facilitate technical implementation we attempted to create a classifier, based on information from a small number of spectral bands and calculation of only relatively simple characteristics, which however provided high sensitivity and specificity of discrimination.

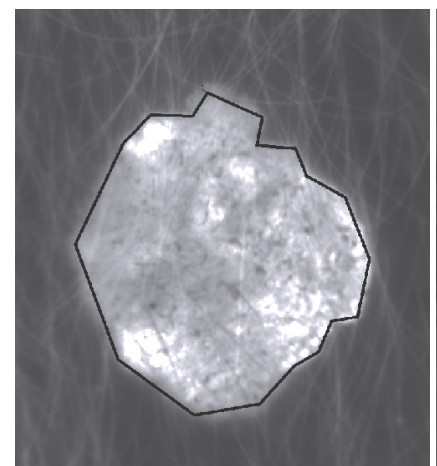

(a)

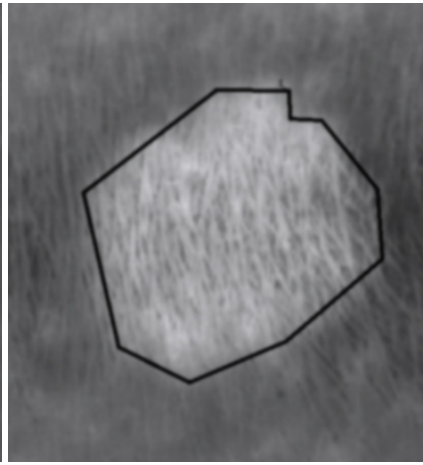

(b)
Fig. 1. Typical acquired optical density images: of melanoma (a), and flat nevus (b) in the spectral band with central wavelength $540 \mathrm{~nm}$. Boundaries of manually segmented lesions shown in black.

Selection of the spectral bands primarily was based on the spectral distribution of mean values and standard deviations 
of pixel optical densities within the analysed skin lesion image. These distribution curves show that value ranges of these characteristics for melanomas and nevi overlap, and, for all characteristics, average values for melanomas are higher than respective values for common nevi. Thus, for classifier building, attention was focused on the spectral bands where minimum values of the melanoma characteristics were closer to maximum values of respective nevi characteristics, i.e. overlap of classes is smaller. Following this approach and taking into account that it is desirable to exploit information from the whole spectral range where the characteristics of melanoma and nevi have meaningful values, design of the first classifier was based on selection of three spectral bands, namely bands 10, 20 and 40 with wavelengths $\lambda_{10}=540 \mathrm{~nm}, \lambda_{20}=640 \mathrm{~nm}$ and $\lambda_{40}=$ $840 \mathrm{~nm}$.

A threshold type classifier was made on the basis of lesion optical characteristics obtained in these spectral bands. Assuming that we have at our disposal $m$ images of melanomas, numbered from 1 to $m$, and $n$ images of nevi numbered from 1 to $n$, let us denote by $\mu_{j}(p)$ the mean optical density of melanoma $p$ image pixels in spectral band $j$, and by $v_{j}(q)$ the mean optical density of nevus $q$ image pixels in spectral band $j$. Likewise let us denote the standard deviation of melanoma $p$ pixel optical density in spectral band $j$ by $s_{j}(p)$, and of nevus $q$ pixel optical density respectively by $\sigma_{j}(q)$. Then let us define:

$$
\begin{cases}a_{j 1}={\underset{\min }{p=1, m}}_{1, m} \mu_{j}(p), & a_{j 2}=\max _{q=1, n} v_{j}(q), \\ b_{j 1}=\min _{p=1, m} s_{j}(p), & b_{j 2}=\max _{q=\overline{1, n}} \sigma_{j}(q) .\end{cases}
$$

As the value ranges of considered characteristics for melanoma and nevus classes overlap and average values for melanomas are higher than respective values for nevi, $a_{j 1}<$ $a_{j 2}$ and $b_{j 1}<b_{j 2}$ for all $j=\overline{1,51}$. Thresholds for the classifier to be designed were found on the basis of following principle: a real number $\Delta, a_{j 1}<\Delta<a_{j 2}$, is chosen to be a threshold for mean values of the band $j$ (and will be denoted by $\Delta_{j}$ ) in such a way that a minimum total volume of outlier sets $U_{j 1}(\Delta)$ and $U_{j 2}(\Delta)$, i.e. $\left|U_{j l}(\Delta)\right|+\left|U_{j 2}(\Delta)\right|$, is provided, where $U_{j 1}(\Delta)=\left\{\mu_{j}(p) \mid \mu_{j}(p)<\Delta\right\}, U_{j 2}(\Delta)=\left\{v_{j}(q) \mid v_{j}(q) \geq\right.$ $\Delta\}$. As the minimum total volume of $U_{j 1}(\Delta)$ and $U_{j 2}(\Delta)$ can be obtained for different values of $\Delta$, any of them can be chosen the initial threshold $\Delta_{j}$.

Thresholds for standard deviations can be defined in the same manner. A real number $\Delta^{\prime}$ will be considered a threshold for standard deviations in band $j$ (and will be denoted by $\left.\Delta_{j}^{\prime}\right)$, if $b_{j 1}<\Delta^{\prime}<b_{j 2}$ and value of $\left|V_{j 1}\left(\Delta^{\prime}\right)\right|+$ $\left|V_{j 2}\left(\Delta^{\prime}\right)\right|$ is minimal in comparison with other possible cases of $\Delta^{\prime}$, where $V_{j 1}\left(\Delta^{\prime}\right)=\left\{s_{j}(p) \mid s_{j}(p)<\Delta^{\prime}\right\}, V_{j 2}\left(\Delta^{\prime}\right)=\left\{\sigma_{j}(q) \mid\right.$ $\left.\sigma_{j}(q) \geq \Delta^{\prime}\right\}$.

Threshold values obtained in accordance with the described procedure are shown in Fig. 2. It can be noticed that location of particular threshold $\Delta_{j}$ between the values $a_{j 1}$ and $a_{j 2}$ (resp., location of $\Delta_{j}^{\prime}$ between $b_{j 1}$ and $b_{j 2}$ ) does not follow a noticeable regularity or relationship with particular spectral regions; and therefore application of the proposed procedure is justified.

Obviously, for needs of our classifier, each multispectral image of the considered skin lesion can be represented by a vector $\left(\alpha_{10}, \alpha_{20}, \alpha_{40}, \beta_{10}, \beta_{20}, \beta_{40}\right)$, where $\alpha_{j}, j \in\{10,20,40\}$ is the mean optical density of the lesion pixels for band $j$, but $\beta_{j}, j \in\{10,20,40\}$ is the standard deviation of optical density of the lesion pixels for band $j$. Consequently we can formulate six statements about each image: $\alpha_{10} \geq \Delta_{10}, \alpha_{20} \geq$ $\Delta_{20}, \alpha_{40} \geq \Delta_{40}, \beta_{10} \geq \Delta^{\prime}{ }_{10}, \beta_{20} \geq \Delta^{\prime}{ }_{20}, \beta_{40} \geq \Delta^{\prime}{ }_{40}$. If the statement is true, it is assigned value 1 , otherwise 0 . Let us denote values corresponding to statements $\alpha_{j} \geq \Delta_{j}, j \in\{10,20$, $40\}$ by $I\left(\alpha_{j}\right)$, and values corresponding to statements $\beta_{j} \geq \Delta^{\prime}$ by $I\left(\beta_{j}\right)$. The classification rule could be formulated on the basis of so called majority voting principle where values 1 indicate the presence of melanoma. However, situations are possible where there is no majority. To resolve them, "votes" can be assigned different weights. Mean values are less informative than standard deviation values as they are more influenced by external factors. Therefore statements $I\left(\beta_{j}\right)$ related to standard deviation parameters were assigned higher weights in the classification rule. Then the classification rule of classifier $W_{1}$ can be formulated: the image of the skin lesion of interest is classified as representing melanoma if and only if

$$
\sum_{j \in\{10,20,40\}}\left(\frac{3}{21} I\left(\alpha_{j}\right)+\frac{4}{21} I\left(\beta_{j}\right)\right)>0.5,
$$

otherwise it is classified as representing nevus.

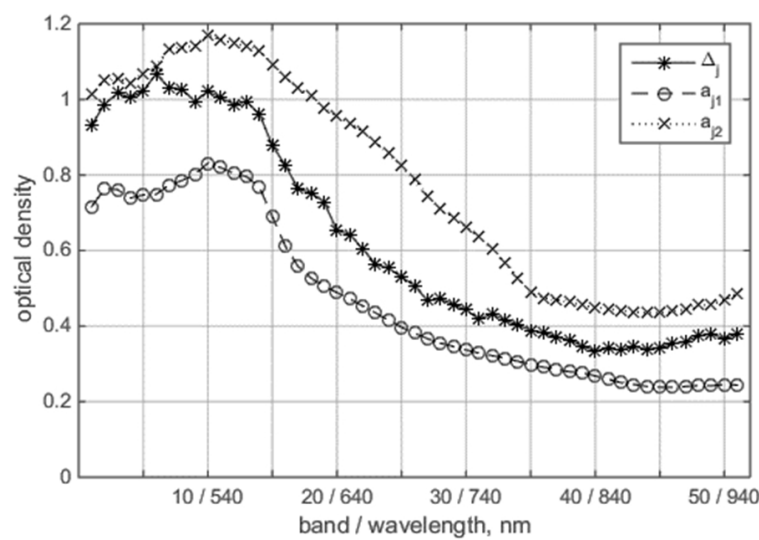

(a)

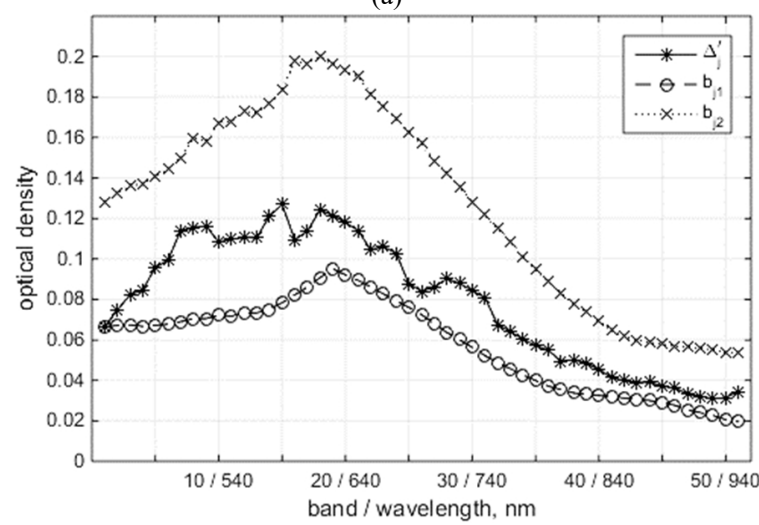

(b)

Fig. 2. Illustration of the procedure for finding threshold values: (a) thresholds for mean values; (b) thresholds for standard deviation values.

Values of weight coefficients may vary as long as they do not change the classification rule considerably. Ratio of the weight coefficients is 1.33 for rule (3). It is easy to check that this ratio can take any value within the interval from 1.1 
to 2.9 without affecting the classifier. For the images at our disposal, classifier based on rule (3) with manual segmentation of lesions in images provided zero false negative (melanoma classified as nevus) and five false positive (nevus classified as melanoma) detection results (four of them were for flat nevi).

\section{SElEction PRINCIPLES OF SPECTRAL BandS}

If the discrimination between melanoma and nevus would be based on information from only one spectral band $j, 1 \leq j$ $\leq 51$, a pair of numbers $\left(g_{j}, h_{j}\right)$ could serve as the measure of its informativeness where:

$$
\left\{\begin{array}{l}
g_{j}=\left|U_{j 1}\left(\Delta_{j}\right)\right|+\left|U_{j 2}\left(\Delta_{j}\right)\right| \\
h_{j}=\left|V_{j 1}\left(\Delta_{j}^{\prime}\right)\right|+\left|V_{j 2}\left(\Delta_{j}^{\prime}\right)\right|
\end{array}\right.
$$

Values of these parameters, obtained for the given set of images for 32 melanomas and 94 nevi, are shown in Fig. 3. Lower values in the graphs correspond to higher informativeness. It can be noticed that values of parameters $h_{j}$ are in general lower so that the assumption about higher informativeness of standard deviation parameters is confirmed.

Two bands from the selected triplet, namely bands 10 and 20, are characterized with good informativeness. It is interesting to note that these bands almost precisely match the bands selected as informative in Diebele et al. [16] on the basis of biomedical arguments. Employing the third band (40) becomes questionable because its informativeness is worse as it is seen in Fig. 3. However, it should be taken into account that the bands to be included in the set should also provide mutually supplementing information. Correlation between pixel values in optical density images obtained from these bands (further shortly referred as correlation between bands) should be assessed to decide on degree of information complementarity of the two bands. Lower correlation between them will indicate that they contain more complementary information.

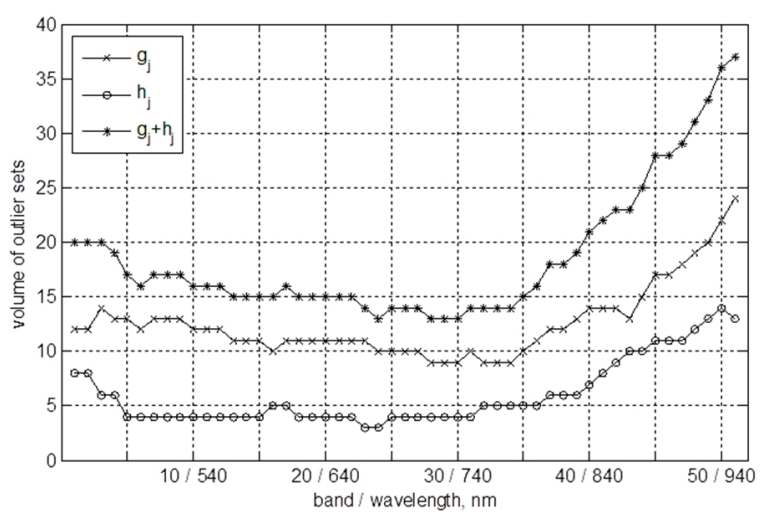

Fig. 3. Informativeness parameters of individual spectral bands

Quantitative assessment of band correlation is associated with certain problems as it is not easy to propose a correlation measure for standard deviation values in different bands. In general, as the optical density spectra of lesions are smooth and slowly changing curves (see [16]), correlation between two spectral bands is higher when the bands are closer. We used the correlation measure between the pairs of spectral bands $(j, k)$ within the set of melanoma lesions as follows

$\operatorname{corr}_{M}(j, k)=\frac{\frac{1}{m-1} \sum_{p=1}^{m}\left(\mu_{j}(p)-\bar{\mu}_{j}\right) \times\left(\mu_{k}(p)-\bar{\mu}_{k}\right)}{s_{j} \times s_{k}}$,

where

$$
\bar{\mu}_{j}=\frac{1}{m} \sum_{p=1}^{m} \mu_{j}(p), \quad \bar{\mu}_{k}=\frac{1}{m} \sum_{p=1}^{m} \mu_{k}(p),
$$

$s_{j}^{2}=\frac{1}{m-1} \sum_{p=1}^{m}\left(\mu_{j}(p)-\bar{\mu}_{j}\right)^{2}, s_{k}^{2}=\frac{1}{m-1} \sum_{p=1}^{m}\left(\mu_{k}(p)-\bar{\mu}_{k}\right)^{2}$.

Analogous measure can be obtained for the set of nevi using the following formula

$$
\operatorname{corr}_{N}(j, k)=\frac{\frac{1}{n-1} \sum_{q=1}^{n}\left(v_{j}(q)-\bar{v}_{j}\right) \times\left(v_{k}(q)-\bar{v}_{k}\right)}{\sigma_{j} \times \sigma_{k}} .
$$

From these separate correlation estimates we can obtain combined correlation measure

$$
\operatorname{corr}(j, k)=\frac{m \times \operatorname{corr}_{M}(j, k)+n \times \operatorname{corr}_{N}(j, k)}{m+n} .
$$

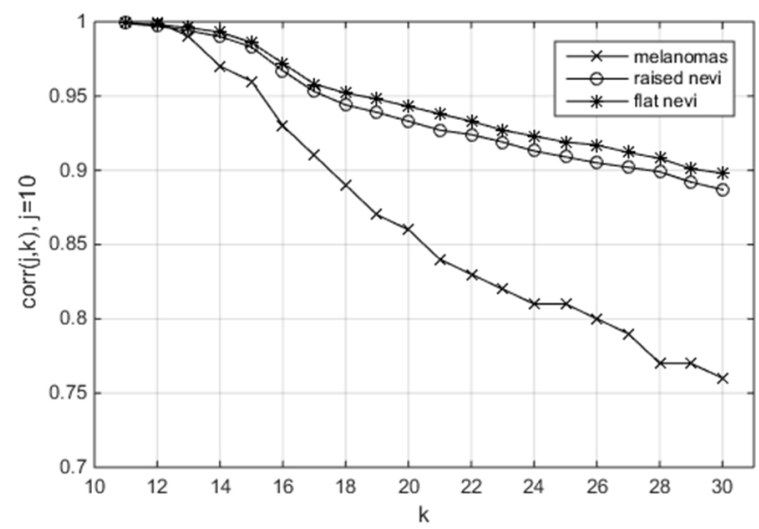

(a)

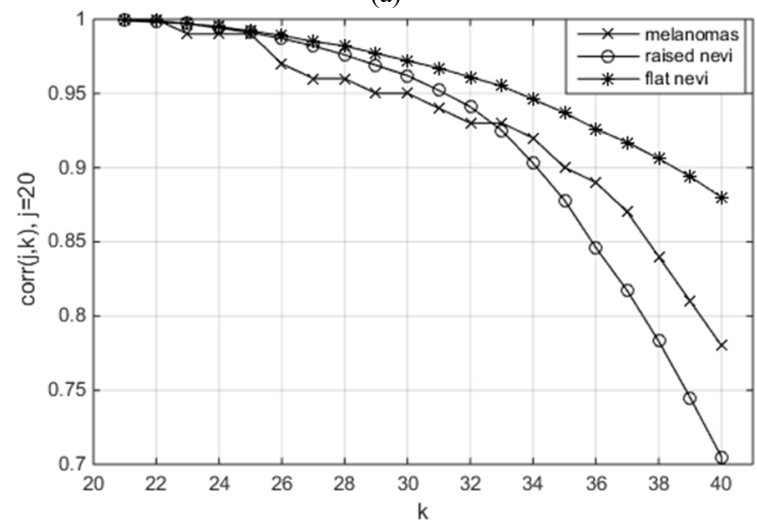

(b)

Fig. 4. Correlation measures between pairs of spectral bands: (a) band 10 with bands from 11 to 30 , (b) band 20 with bands from 21 to 40 .

The number of pairs of spectral bands in our case is $C_{51}{ }^{2}$ $=1275$, however we focused our main attention on analysis of correlation bands 10 and 20 and their neighbours with higher wavelengths. Analysis results are presented in Fig. 4. If the correlation of two bands is positive and close to 1 , 
inclusion of both bands into the same set should be considered unjustified. It can be noticed from Fig. 4(a) that bands from 11 to 15 are highly correlated with band 10 but bands from 17 and above show less correlation with band 10. Similarly, from Fig. 4(b) we see that bands from 21 to 25 are highly correlated with band 20 but bands from 26 and above are less correlated and can provide complementary data. Correlation measure for bands 10 and 20 within the set of melanoma lesions is $\operatorname{corr}_{M}(10,20)=0,86$ so that information from these bands complement each other. The pair of bands $(10,30)$ is even less correlated and provides more substantial complementary information. Low correlation between bands 20 and 40 as seen in Fig. 4(b) may serve as a justification for using the triplet of bands (10, 20, 40).

Mutually complementary information is provided also by the triplet of bands $(10,20,30)$ as the band 30 is far enough from band 20 so that correlation between bands 20 and 30 is weakened. This choice is also justified by the band informativeness shown in Fig. 3. To prepare a classifier based on bands 10,20 and 30 , thresholds $\Delta_{30}$ and $\Delta^{\prime}{ }_{30}$ were calculated in a similar way as $\Delta_{10}, \Delta_{10}^{\prime}, \Delta_{20}$ and $\Delta^{\prime}{ }_{20}$ and the classification rule analogous to (3) was used, where the band 40 was replaced with band 30 . Using this classifier, zero false negative and four false positive melanoma detections (three of them for flat nevi) were obtained. From the results, it can be concluded that usage of the triplet of spectral bands $(10,20,30)$ for melanoma detection is favourable in comparison with triplet $(10,20,40)$, apparently due to higher informativeness of band 30 compared to band 40 .

\section{Stability OF THE ClassifiER}

Considering technical implementation possibilities of the device exploiting the proposed classifier, the following question is raised: shall the precision characteristics be affected by possible widening of the bandwidths of chosen spectral bands due to limited spectral resolution of used equipment so that in reality each used spectral band could be widened and include information from neighbouring bands? To answer this question at least partly, initial classification rule was changed. Characteristics $\mu_{j}(p)$ and $s_{j}(p)$ for a spectral band $j$ for melanoma $p$ were replaced by characteristics of a random variable $Y_{j}(p)=0.25 X_{j-1}(p)+0.5 X_{j}(p)+0.25 X_{j+1}(p)$, namely mean value

$$
\begin{gathered}
E Y_{j}(p)=\mu_{j}^{\prime}(p)= \\
=0.25 \mu_{j-1}(p)+0.5 \mu_{j}(p)+0.25 \mu_{j+1}(p),
\end{gathered}
$$

and standard deviation

$$
\sqrt{E\left(Y_{j}(p)-E Y_{j}(p)\right)^{2}}=s_{j}^{\prime}(p)
$$

where $X_{j-1}(p), \quad X_{j}(p), \quad X_{j+1}(p), \quad$ are random variables representing distribution of image pixels' OD for melanoma $p$.

If the number of lesion pixels in the image of melanoma $p$ is equal to $m_{p}$ and optical density of pixel $s$ in band $j$ is equal to $x_{s}(p, j)$, OD in band $j-1$ is equal to $x_{s}(p, j-1)$, and OD in band $j+1$ is equal to $x_{s}(p, j+1)$, then we will obtain the following estimates of $\mu_{j}^{\prime}(p)$ and $s_{j}^{\prime}(p)$ :

$$
\begin{gathered}
\mu_{j}^{\prime}(p)=\frac{1}{m_{p}} \sum_{s=1}^{m_{p}}\left(\begin{array}{l}
0.25 x_{s}(p, j-1)+ \\
+0.5 x_{s}(p, j)+0.25 x_{s}(p, j+1)
\end{array}\right) \\
s_{j}^{\prime}(p)=\sqrt{\frac{1}{m_{p}-1} \sum_{s=1}^{m_{p}}\left(\begin{array}{l}
\left.0.25\left(x_{s}(p, j-1)-\mu_{j-1}(p)\right)+\right)^{2} \\
+0.5\left(x_{s}(p, j)-\mu_{j}(p)\right)+ \\
+0.25\left(x_{s}(p, j+1)-\mu_{j+1}(p)\right)
\end{array}\right)},
\end{gathered}
$$

If the image of nevus $q$ is considered, and OD distribution of its pixels in band $j$ is represented by the random variable $X_{j}(q)$, OD in band $j-1$ represented by $X_{j-1}(q)$, and OD in band $j+1$ represented by $X_{j+l}(q)$, then $v_{j}(q)$ and $\sigma_{j}(q)$ are replaced by the characteristics of the random variable $Y_{j}(q)=0.25 X_{j-1}(q)+0.5 X_{j}(q)+0.25 X_{j+1}(q), \quad$ namely its mean value

$$
\begin{gathered}
E Y_{j}(q)=v_{j}^{\prime}(p)= \\
=0.25 v_{j-1}(q)+0.5 v_{j}(q)+0.25 v_{j+1}(q),
\end{gathered}
$$

and standard deviation

$$
\sqrt{E\left(Y_{j}(q)-E Y_{j}(q)\right)^{2}}=\sigma_{j}^{\prime}(q)
$$

If the classifier based on triplet of bands $(10,20,40)$, using rule (3) and threshold values $\Delta_{j}$ and $\Delta_{j}^{\prime}$ calculated above is denoted by $W_{1}$, a classifier based on expanded bands (keeping the same rule and threshold values) can be denoted by $W_{1}^{\prime}$. Analogously, corresponding classifiers based on triplet of bands $(10,20,30)$ can be denoted by $W_{2}$ and $W_{2}^{\prime}$.

Application of classifier $W_{1}^{\prime}$ for images at our disposal provided zero false negative results for melanoma and one false positive result for flat nevus. Classifier $W_{2}^{\prime}$ provided identical results. The same conditions of the experiments were used, namely manual segmentation of lesions and threshold values $\Delta_{j}$ and $\Delta_{j}^{\prime}$ calculated before.

\section{DISCUSSION}

Taking into account that the estimate of the correlation coefficient for the pair of bands $(10,30)$ within the set of melanomas is 0.76 , it is interesting to consider a classifier based on these two bands only. Using the same threshold levels as for classifier based on bands (10, 20, 30), classification rule is formulated as follows: an image is classified as representing melanoma if and only if

$$
\sum_{j \in\{10,30\}}\left(0.24 I\left(\alpha_{j}\right)+0.26 I\left(\beta_{j}\right)\right) \geq 0.48
$$

Let us denote that classifier by $W_{3}$. If we combine the central bands with neighbouring ones using the weighting coefficients $0.25,0.5,0.25$, then the classifier $W_{3}^{\prime}$ is obtained. Application of these classifiers to available images of melanomas and nevi provided the following results: using 
the classifier $W_{3}$, zero false negative and 10 false positive (from them, 6 for flat nevi) classifications were obtained; using the classifier $W_{3}^{\prime}$, there were zero false negative and 6 false positive (from them, 2 for flat nevi) results.

Note that changing of threshold for rules from 0.5 to 0.48 does not change the properties of classifiers $W_{1}, W_{1}^{\prime}, W_{2}$ and $W_{2}^{\prime}$.

Discrimination between melanomas and nevi can be performed also in various other ways, e.g. based on the design of a support vector machine (SVM). This option was used by Quinzán et al. [20]. Although the result they obtained (classifier based on usage of 9 spectral bands) has provided considerable quality in terms of sensitivity $(=1)$ and specificity $(=0.72)$, nevertheless it is not the last word in that problem area, namely development of melanoma discriminators based on information from few spectral bands. In our research, number of spectral bands was reduced to 3 without sacrificing quality of the classifier. This difference matters when practical implementation is considered.

To exploit the experimental application results of classifiers $W_{1}, W_{1}^{\prime}, W_{2}$ and $W_{2}^{\prime}$ for statistical estimation of sensitivity and specificity, we have to accept the following postulate idealizing the real situation: application of a particular classifier to analysis of a particular melanoma (resp., particular nevus) is characterized by one and the same error probability $p_{M}$ (resp., $\left.q_{N}\right)$. In this case, statistical estimate of sensitivity (resp. specificity) for a given classifier could be calculated using the maximum likelihood method. From the results of our experiments, sensitivity of the classifier cannot be estimated on the basis of the maximum likelihood method because there is no maximum point of the function $p_{M}^{32}$, defined in the interval $[0,1]$. At the same time, note that such classification result (all images of melanomas classified correctly) can be achieved with rather high probability also if the true sensitivity value is 0.99 . Probability of such event is 0.7249 . However, true sensitivity cannot be less than 0.85 as the probability of such event is lower than 0.0055 .

In general, if there is sufficient amount of data available, it is desirable to test performance of the classifier with test data set that is different from the data set used for its design. However, it should be mentioned that, in order to have a statistically significant testing result, test dataset should be a representative sample from the melanoma and nevi universe. If that condition is not met, value of the testing result will be questionable regardless of the fact that the test set is different from the design set. As the images used in the study were collected over a prolonged period of time from different patients, and the number of images in sample sets from each universe is sufficiently high (especially for the set of nevi), they can be qualified as representative for the local population. To check robustness of the classifier $W_{2}$, the following experimental procedure was used: the data set of 32 melanoma images (resp. 94 nevus images) was split in a random way into two subsets containing 16 melanoma images (resp. 47 nevus images), one of them used for calculation of thresholds $\Delta_{j}$ and $\Delta_{j}^{\prime}$ whereas the other used as a test set in the classification experiment based on the rule (3) and bands $(10,20,30)$. This procedure was repeated 20 times; number of false negative and false positive melanoma detections is illustrated in Fig. 5.

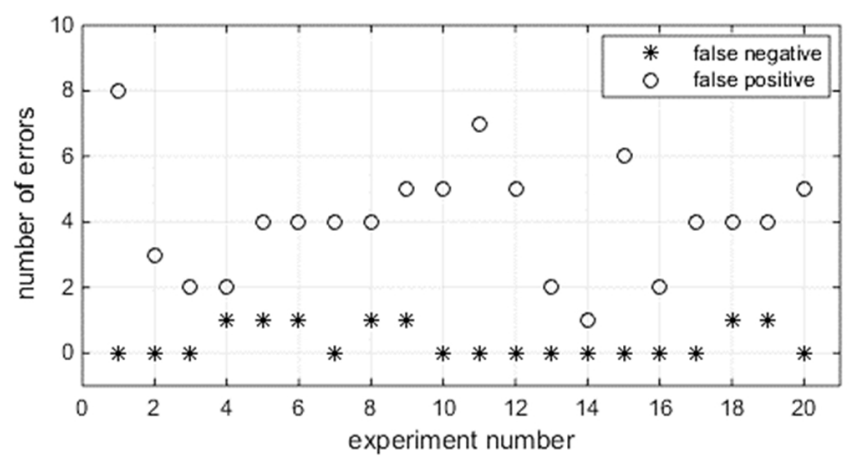

Fig. 5. Experimental testing results of classifier $W_{2}$.

It is noticed that the number of classification errors remain low for all experiments. Further investigations could be useful to validate applicability of the proposed approach to different types of skin.

Sensitivity and specificity values obtained in a particular experiment are only statistical point estimates characterizing a classifier with certain probability. For example, if a classifier correctly classifies 7 melanomas as in [20], then the estimate of sensitivity equal to 1 is questionable because the same experimental result with sufficiently high probability could be obtained also if the true sensitivity value would be 0.96 . This probability is higher than 0.75 . We can speak about $100 \%$ sensitivity only in the case when such characteristic is justified theoretically on the basis of certain physical parameters.

New techniques and multidisciplinary approaches are of interest for improved detection of melanoma [5]. Diffuse reflectance and fluorescence imaging could be considered as complementary methods for skin diagnostics [26]. It should be mentioned that the proposed approach also facilitates fusion of image data acquired using different technologies. As far as statistical characteristics are obtained separately for each image related with the particular spectral band, there are no restrictions to how this image is acquired. For example, a fluorescence image of a skin lesion acquired exploiting corresponding data acquisition technology could be used to calculate mean value and standard deviation of a lesion and related statements can participate in classification together with statements formulated from lesion reflectance images. That may facilitate application of the proposed approach to different diagnostic tasks in biomedicine.

Development of low cost diagnostic devices for primary screening of skin lesions would improve accessibility of early diagnosis that is important especially for developing countries and Central and Eastern Europe [4]. Smartphones become more advanced and available and find applications in skin diagnostics; for example, HandyScope from FotoFinder Systems [27] is a dermoscope compatible with a smartphone. Proposed simplified approach based on only few spectral bands could be implemented as a simple device or a connection kit for a smartphone.

\section{CONCLUSIONS}

The proposed method for discrimination of melanoma from benign nevi is based on quantifiable spectral properties and characterized by high sensitivity and specificity statistical point estimates whereas its possible technical 
implementation is fairly simple. That would require hardware for obtaining images only in three spectral bands within the region of visible light where the low cost light sources and digital photo sensors are widely available. Involved image processing would include image segmentation and rather simple calculation of statistical characteristics of pixel values for the lesion considered. Related software can be prepared for the connected general purpose device used also for visualization and storage of acquired images together with melanoma detection results and corresponding recommendations. With such approach, a low cost device could be proposed to assist primary care physicians in making decision about the necessity to visit a specialized clinic and thus help in early diagnostics of cutaneous melanoma.

\section{ACKNOWLEDGMENT}

The authors express their gratitude to the Institute of Atomic Physics and Spectroscopy (University of Latvia) and especially to Ilze Lihacova for the spectral data of pigmented skin lesions. The authors would like to thank Dr. Peteris Treijs for his valuable suggestions as well as Madis Menke for his assistance.

\section{REFERENCES}

[1] American Cancer Society. Cancer Facts \& Figures 2014. [Online]. Available: http://www.cancer.org/acs/groups/content/@research/ documents/webcontent/acspc-042151.pdf

[2] K. Azarjana, A. Ozola, D. Ruklisa, I. Cema, A. Rivosh, A. Azaryan, D. Pjanova, "Melanoma epidemiology, prognosis and trends in Latvia", J. Eur. Acad. Dermatol. Venereol., vol. 27, no. 11, pp. 13521359, 2013. [Online]. Available: http://dx.doi.org/10.1111/jdv.12007

[3] J. Ferlay, E. Steliarova-Foucher, J. Lortet-Tieulent, S. Rosso, J. W. W. Coebergh, H. Comber, D. Forman, F. Bray, "Cancer incidence and mortality patterns in Europe: estimates for 40 countries in 2012", Eur. J. Cancer, vol. 49, no. 6, pp. 1374-403, 2013. [Online]. Available: http://dx.doi.org/10.1016/j.ejca.2012.12.027

[4] A. M. Forsea, V. Del Marmo, E. de Vries, E. E. Bailey, A. C. Geller, "Melanoma incidence and mortality in Europe: new estimates, persistent disparities", Br. J. Dermatol., vol. 167, no. 5, pp. 1124-30, 2012. [Online]. Available: http://dx.doi.org/10.1111/j.13652133.2012.11125.x

[5] D. S. Rigel, J. Russak, R. Friedman, "The evolution of melanoma diagnosis: 25 years beyond the ABCDs", CA-Cancer J. Clin., vol. 60, no. 5, pp. 301-316, 2010. [Online]. Available: http://dx.doi.org/10.3322/caac.20074

[6] F. Nachbar, W. Stolz, T. Merkele, A. B. Cognetta, T. Vogt, M. Landthaler, P. Bilek, O. Braun-Falco, G. Plewig, "The ABCD rule of dermatoscopy: high prospective value in the diagnosis of doubtful melanocytic skin lesions", J. Am. Acad. Dermatol., vol. 30, no. 4, pp. 551-559, 1994. [Online]. Available: http://dx.doi.org/10.1016/ S0190-9622(94)70061-3

[7] R. Amelard, J. Glaister, A. Wong, D. A. Clausi, "High-level intuitive features (HLIFs) for intuitive skin lesion description", IEEE Trans. Biomed. Eng., vol. 62, no. 3, pp. 820-831, 2015. [Online]. Available: http://dx.doi.org/10.1109/TBME.2014.2365518

[8] D. L. Farkas, D. Becker, "Applications of spectral imaging: detection and analysis of human melanoma and its precursors", Pigm. Cell Res. vol. 14, no. 1, pp. 2-8, 2001. [Online]. Available: http://dx.doi.org/10. 1034/j.1600-0749.2001.140102.x

[9] J. Zhang, C. I. Chang, S. J. Miller, K. A. Kang, "A feasibility study of multispectral image analysis of skin tumors", Biomed. Instrum. Technol., vol. 34, no. 4, pp. 275-282, 2000.

[10] B. Farina, C. Bartoli, A. Bono, A. Colombo, M. Lualdi, G. Tragni, R. Marchesini, B. Farina, C. Bartoli, A. Bono, A. Colombo, M. Lualdi, G. Tragni, "Multispectral imaging approach in the diagnosis of cutaneous melanoma: potentiality and limits", Phys. Med. Biol., vol. 45, no. 5, pp. 1243-1254, 2000. [Online]. Available: http://dx.doi.org/10.1088/0031-9155/45/5/312

[11] S. Tomatis, M. Carrara, A. Bono, C. Bartoli, M. Lualdi, G. Tragni, A. Colombo, R. Marchesini, "Automated melanoma detection with a novel multispectral imaging system: results of a prospective study", Phys. Med. Biol., vol. 50, no. 8, pp. 1675-1687, 2005. [Online] Available: http://dx.doi.org/10.1088/0031-9155/50/8/004

[12] R. Marchesini, A. Bono, S. Tomatis, C. Bartoli, A. Colombo, M Lualdi, M. Carrara, "In vivo evaluation of melanoma thickness by multispectral imaging and an artificial neural network. A retrospective study on 250 cases of cutaneous melanoma", Tumori, vol. 93, no. 2 , pp. 170-177, 2007.

[13] T. Nagaoka, Y. Kiyohara, H. Koga, A. Nakamura, T. Saida, T. Sota, "Modification of a melanoma discrimination index derived from hyperspectral data: a clinical trial conducted in 2 centers between March 2011 and December 2013", Skin Res. Technol., (to be published). [Online]. Available: http://dx.doi.org/10.1111/srt.12188

[14] F. Vasefi, N. MacKinnon, R. B. Saager, A. J. Durkin, R. Chave, E. H. Lindsley, D. L. Farkas, "Polarization-sensitive hyperspectral imaging in vivo: a multimode dermoscope for skin analysis", Sci. Rep., vol. 4 , 2014.

[15] I. Kuzmina, I. Diebele, D. Jakovels, J. Spigulis, L. Valeine, J. Kapostinsh, A. Berzina, "Towards non-contact skin melanoma selection by multi-spectral imaging analysis", J. Biomed. Opt., vol. 16, no. 6, 2011. [Online]. Available: http://dx.doi.org/10.1117/1.3584846

[16] I. Diebele, I. Kuzmina, A. Lihachev, J. Kapostinsh, A. Derjabo, L.Valeine, J. Spigulis, "Clinical evaluation of melanomas and common nevi by spectral imaging", Biomed. Opt. Express, vol. 3, no. 3, 2012. [Online]. Available: http://dx.doi.org/10.1364 BOE.3.000467

[17] R. Jolivot, P. Vabres, F. Marzani, "Reconstruction of hyperspectral cutaneous data from an artificial neural network-based multispectral imaging system", Comput. Med. Imaging Graph., vol. 35, no. 2, pp. 85-88, 2011. [Online]. Available: http://dx.doi.org/10.1016/j. compmedimag.2010.07.001

[18] M. Elbaum, A. W. Kopf, H. S. Jr. Rabinovitz, R. G. Langley, H. Kamino, M. C. Jr. Mihm, A. J. Sober, G. L. Peck, A. Bogdan, D. Gutkowicz-Krusin, M. Greenebaum, S. Keem, M. Oliviero, S. Wang, "Automatic differentiation of melanoma from melanocytic nevi with multispectral digital dermoscopy: a feasibility study", $J$ Am. Acad. Dermatol., vol. 44, no. 2, pp. 207-218, 2001. [Online] Available: $\mathrm{http}: / / \mathrm{dx}$. doi.org/10.1067/mjd.2001.110395

[19] M. Moncrieff, S. Cotton, E. Claridge, P. Hall, "Spectrophotometric intracutaneous analysis: a new technique for imaging pigmented skin lesions", Br. J. Dermatol., vol. 146, no. 3, pp. 448-457, 2002. [Online]. Available: http://dx.doi.org/10.1046/j.1365-2133.2002 04569.x

[20] I. Quinzan, J. M. Sotoca, P. Latorre-Carmona, F. Pla, P. GarciaSevilla, E. Boldo, "Band selection in spectral imaging for noninvasive melanoma diagnosis", Biomed. Opt. Express, vol. 4, no. 4, pp. 514-519, 2013. [Online]. Available: http://dx.doi.org/ 10.1364/BOE.4.000514

[21] A. P. Dhawan, B. D'Alessandro, S. Patwardhan, N. Mullani, "Multispectral optical imaging of skin-lesions for detection of malignant melanomas", in Conf. Proc. IEEE Eng. Med. Biol. Soc., 2009, pp. 5352-5355. [Online]. Available: http://dx.doi.org 10.1109 /iembs.2009.5334045

[22] D. Kapsokalyvas, N. Bruscino, D. Alfieri, V. de Giorgi, G. Cannarozzo, R. Cicchi, D. Massi, N. Pimpinelli, F. S. Pavone, "Spectral morphological analysis of skin lesions with a polarization multispectral dermoscope", Opt. Express, vol. 21, no. 4, pp. 4826$4840,2013 . \quad$ [Online]. Available: http://dx.doi.org/10.1364/ OE. 21.004826

[23] A. Lorencs, I. Mednieks, J. Sinica-Sinavskis, "Simplified Classification of Multispectral Image Fragments", Elektronika ir Elektrotechnika, vol. 20, no. 6, pp. 136-139, 2014. [Online]. Available: $\mathrm{http}: / / \mathrm{dx}$.doi.org/10.5755/j01.eee.20.6.7286

[24] S. G. Demos, R. R. Alfano, "Optical polarization imaging", Appl. Opt., vol. 36, no. 1, pp. $150-155$, 1997. [Online]. Available: http://dx.doi.org/10.1364/AO.36.000150

[25] M. E. Celebi, H. Iyatomi, G. Schaefer, W. V. Stoecker, "Lesion border detection in dermoscopy images", Comput. Med. Imaging Graph., vol. 33, no. 2, pp. 148-153, 2009. [Online]. Available: http://dx.doi.org/10.1016/j.compmedimag.2008.11.002

[26] Y. Bae, J. S. Nelson, B. Jung, "Multimodal facial color imaging modality for objective analysis of skin lesions", J. Biomed. Opt., vol. 13, no. 6, 2008. [Online]. Available: http://dx.doi.org/10.1117/ 1.3006056

[27] W. F. Zha, W. M. Song, J. J. Ai, A. E. Xu, "Mobile connected dermatoscope and confocal laser scanning microscope: a useful combination applied in facial simple sensitive skin", Int. J. Cosmet. Sci., vol. 34, no. 4, pp. 318-321, 2012. [Online]. Available: http://dx.doi.org/10.1111/j.1468-2494.2012.00726.x 